\title{
A New Low Complexity Optimal Decoding Method for MIMO Systems
}

\author{
Mohammad Ali Maddah-Ali ${ }^{\dagger}$, Amir K. Khandani ${ }^{\dagger}$, and Wen Tong ${ }^{\dagger \dagger}$ \\ ${ }^{\dagger}$ Coding and Signal Transmission Lab., Elec. \& Comp. Eng. dept., Univ. of Waterloo \\ http://cst.uwaterloo.ca, Email: \{mohammad,khandani\}@ cst.uwaterloo.ca \\ ${ }^{\dagger \dagger}$ Nortel Networks, Nepean, ON, Canada, K2G 6J8
}

\begin{abstract}
A method is proposed to reduce the complexity of ML decoding for MIMO systems based on PSK constellation with two transmit antennas. We use the feature that the points of a PSK constellation form a finite group under complex multiplication to substantially reduce the decoding complexity. The proposed method of decoding is used for both BLAST systems and a new full-rate full-diversity space-time code. The proposed space-time code offers a high coding advantage and at the same time allows for a simple decoding algorithm.
\end{abstract}

\section{INTRODUCTION}

Nowadays, demand for reliable high data rate wireless communication is increasingly growing. Multiple Input-Multiple Output (MIMO) systems, corresponding to using multiple antennas at both sides of the wireless link, are widely recognized as a solution for this demand. There are two methods of signalling over MIMO channels: BLAST [1] and space-time coding [2].

BLAST is a well-known method for high data rate communication over MIMO channels [1]. Although some suboptimal decoding methods such as ZF-BLAST and MMSE-BLAST are introduced in [1] [3], finding a reduced complexity optimal BLAST decoding algorithm is still an open problem. In this paper a new optimal decoding method for a BLAST system with for two transmit antennas is presented. The complexity of this method is considerably less than the complexity of the exhaustive search. At first, we present our decoding method for an $r$-PSK constellation, while later, the method is generalized to other symmetric constellations like QAM.

On the other hand, space-time coding is a well-known method to achieve space diversity as well as coding gain over a MIMO system. Finding a low complexity optimal decoding for space-time codes is a challenging problem. Some researchers have addressed this problem by imposing some restrictions on the code structure which facilitates the decoding operation at the cost of a reduced coding gain. [2] [4].

In this paper, a new structure for full-rate full-diversity space time code is introduced. This code is a superposition of two families of matrices, where each of them forms a finite group under complex multiplication. We show that the decoding formulation derived for BLAST systems can be also applied for the decoding of the proposed space-time scheme. On the other hand, we will see that the coding advantage of this structure is very high.

\section{FORMULATION}

In slow flat fading environment, a MIMO channel with 2 transmit and $N$ receive antennas is modelled by

$$
y=\sqrt{\frac{\rho}{2}} H x+v
$$

where $y \in \mathcal{C}^{N \times 1}$ denotes the received vector and $x \in \mathcal{C}^{2 \times 1}$ denotes the transmitted vector, $H \in \mathcal{C}^{N \times 2}$ denotes the channel matrix, and $v \in \mathcal{C}^{N \times 1}$ denotes additive, spatially and temporally i.i.d white noise with complex gaussian distribution. Transmitted vector $x$ and noise vector $v$ are normalized such that $\rho$ is the Signal to Noise $\operatorname{Ratio}(\mathrm{SNR})$ at each receive antennas.

\section{DECODING METHOD}

In the following, we first present the proposed decoding method for an $r$-PSK constellation, while later, the method is generalized for other symmetric constellations.

In $r$-PSK modulation, transmitted vector is as follows,

$$
x_{m, n}=\left[\theta^{m}, \theta^{n}\right]^{T} \quad \theta=\exp \left(j \frac{2 \pi}{r}\right)
$$

where $m$ and $n(0 \leq m, n \leq r-1)$, are two transmitted symbols.

To formulate the $M L$ decoding, we have,

$$
P\left(y \mid H, x_{m, n}\right)=\frac{1}{\pi^{N T}} \exp \left(-d_{m n}^{2}\right)
$$

where,

$$
d_{m n}^{2}=\left(y-\sqrt{\frac{\rho}{2}} H x_{m, n}\right)^{*}\left(y-\sqrt{\frac{\rho}{2}} H x_{m, n}\right)
$$

The goal of ML decoding is to find $m$ and $n$ to maximize $P\left(y \mid H, x_{m, n}\right)$ or minimize $d_{m n}^{2}$. The straight-forward approach for ML decoding is to calculate different values of $d_{m n}^{2}$ for all possible values of $m$ and $n$, and find the minimum value of $d_{m n}^{2}$ using an exhaustive search. It is clear that the complexity of such an exhaustive search is very high.

Let us define $K, f(n), g(m)$ and $h(m-n)$ as:

$$
\begin{gathered}
K=y^{*} y+\frac{\rho}{2}\left(h_{1}^{*} h_{1}+h_{2}^{*} h_{2}\right) \\
f(m)=-\sqrt{\frac{\rho}{2}}\left(y^{*} h_{1} \theta^{m}+h_{1}^{*} y \theta^{-m}\right)
\end{gathered}
$$




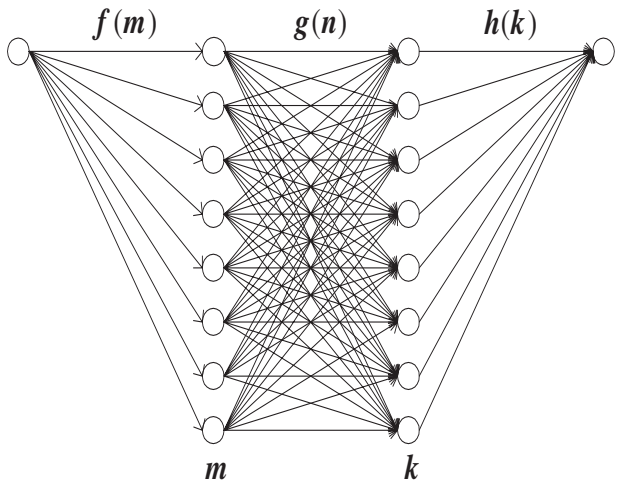

Fig. 1. Trellis constructed based on code structure

$$
\begin{gathered}
g(n)=-\sqrt{\frac{\rho}{2}}\left(y^{*} h_{2} \theta^{n}+h_{2}^{*} y \theta^{-n}\right) \\
h(m-n)=\frac{\rho}{2}\left(h_{2}^{*} h_{1} \theta^{(m-n)}+h_{1}^{*} h_{2} \theta^{-(m-n)}\right)
\end{gathered}
$$

where $h_{1}$ and $h_{2}$ are the first and second columns of channel matrix $H$.

Using these notations, it is easy to show that,

$$
d_{m n}^{2}=K+f(m)+g(n)+h(m-n)
$$

Considering that $\theta^{r}=\theta^{0}=1$, it is easy to prove that $h(m-n)=h(m-n \bmod r)$. Thus, there are $r$ different values for each of $f(m), g(n)$, and $h(m-n)$ where $0 \leq$ $m \leq r-1$ and $0 \leq n \leq r-1$.

Ignoring the constant part $K$, for minimizing $f(m)+g(n)+$ $h(m-n)$, we can use Viterbi algorithm over the trellis structure shown as an example for the case of 8-PSK in Fig. 1. In this figure, $k=[(m-n) \bmod r]$.

Another effective ML decoding method with less complexity is as follows: Let us sort $f(m)$ and $g(m)$ in the increasing order and specify the corresponding arguments as $m_{0}, m_{1} \ldots m_{r-1}$ and $n_{0}, n_{1} \ldots n_{r-1}$, i.e.,

$$
f\left(m_{0}\right) \leq f\left(m_{1}\right) \leq \ldots \leq f\left(m_{r-1}\right)
$$

and,

$$
g\left(n_{0}\right) \leq g\left(n_{1}\right) \leq \ldots \leq g\left(n_{r-1}\right)
$$

We define two sets, named the potential set and the final set. Each set has $r$ entries corresponding to different values of $[(m-n) \bmod r]$. The $k^{\text {th }}$ entry of the final set, $0 \leq k \leq$ $r-1$, is the best pair of $(m, n)$ in terms of the minimization of $f(m)+g(n)$ such that $k=[(m-n) \bmod r]$. The $k^{\text {th }}$ entry of the potential set, $0 \leq k \leq r-1$, is the best pair of $(m, n)$ in terms of the minimization of $f(m)+g(n)$ such that $k=[(m-n) \bmod r]$ until then, and for those values of $k$ that are not yet in the final set. The final set will be gradually filled using the following algorithm:

For $0 \leq \alpha \leq 2(r-1)$, starting from $\alpha=0$,

1) Find $S_{\alpha}=\{(i, j) \mid i+j=\alpha, 0 \leq i \leq r-1,0 \leq j \leq$ $r-1\}$.

2) Find $(i, j) \in S_{\alpha}$ that minimize $f\left(m_{i}\right)+g\left(n_{j}\right)$.
3) Set $k=\left[\left(m_{i}-n_{j}\right) \bmod r\right]$. Compare $\left(m_{i}, n_{j}\right)$ with the pair in $k^{\text {th }}$ row of potential set, if any, in terms of $f(m)+g(n)$ and put the best one in $k^{\text {th }}$ row of final set (if $k^{\text {th }}$ row of final set is not filled yet).

4) For other pairs of $(i, j) \in S_{\alpha}$, compute $k=\left[\left(m_{i}-n_{j}\right)\right.$ $\bmod r]$ and compare $f\left(m_{i}\right)+g\left(n_{j}\right)$ with the related value in the $k^{\text {th }}$ row of the potential set. Put the better of these two values in the $k^{\text {th }}$ row of the potential set.

5) If the final set is not filled yet, set $\alpha \leftarrow \alpha+1$ and go to step 1 .

6) If the final set is not filled yet and $\alpha<2(r-1)$, set $\alpha \leftarrow \alpha+1$ and go to the step 1 , else fill the empty rows of final set with potential set.

7) Compute $f(m)+g(n)+h(m-n)$ for pairs in the final set and select the pair that minimize $f(m)+g(n)+h(m-n)$.

Simulations show that the truncation of the algorithm in $\alpha=r-1$ does not considerably affect the code performance (probability of the additional error is less than $10^{-6}$ ).

The idea behind the algorithm is as follows: If $(i, j) \in S_{\alpha}$ minimize $f\left(m_{i}\right)+g\left(n_{j}\right)$ and $k=\left[\left(m_{i}-n_{j}\right) \bmod r\right]$, then there is no $\left(i^{\prime}, j^{\prime}\right) \in S_{\alpha^{\prime}},\left(\alpha<\alpha^{\prime}\right)$ such that $k=\left[\left(m_{i^{\prime}}-n_{j^{\prime}}\right)\right.$ $\bmod r]$ and $f\left(m_{i}\right)+g\left(n_{j}\right)>f\left(m_{i^{\prime}}\right)+g\left(n_{j^{\prime}}\right)$.

In addition to PSK constellation (closed under addition of phases), similar ideas can be applied to QAM constellations. In the case of QAM, it is easy to break down the constellation into a number of PSK-type subsets such that each subset is closed under addition of phases. In this case, in the Cartesian product of such QAM constellations (corresponding to different antennas), we obtain a number of elements that can be decoded separately using the above techniques (each such element is the Cartesian product of some PSK-type subsets where each subset corresponds to a different antenna). For example, in 16QAM constellation, we can classify all the points into 4 individual sets $\mathrm{A}, \mathrm{B}, \mathrm{C}$, and $\mathrm{D}$ as shown in Fig. 2. In this case, we can rewrite the transmitted vector as follows,

$$
x=\left[\alpha_{i} \theta^{m}, \alpha_{j} \theta^{n}\right]^{T} \quad \theta=\exp \left(j \frac{2 \pi}{r}\right)
$$

where

$$
0 \leq i, j, m, n \leq 3
$$

and $\alpha_{i}$ and $\alpha_{j}$ determine which subset is selected and, $m$ and $n$ determine which points in each subset are selected. Based on this formulation, we can use the same decoding algorithm, after some small modifications.

\section{Complexity}

In this part, we review the complexity of the proposed decoding method and compare it with the complexity of the exhaustive search. In MIMO systems with $N$ receive antennas and $r$-PSK constellation, it is easy to show that the complexity of the exhaustive search is equal to $20 N r^{2}$. On the other hand, the complexity of the proposed method is $1.5 r^{2}+24 r N$ that is considerably less the complexity of the exhaustive search. For typical values of $r$ and $N$, the complexity of proposed method 


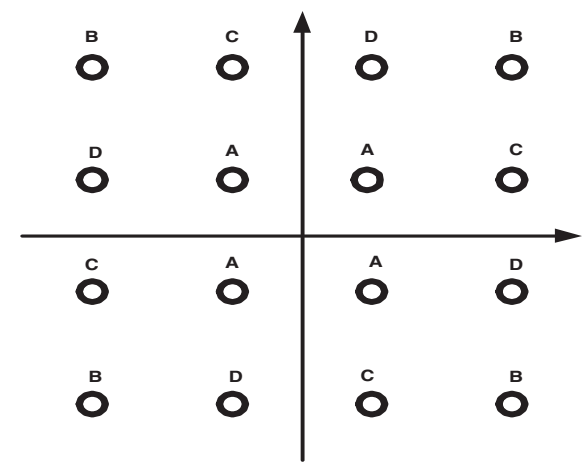

Fig. 2. Classification of 16QAM into 4 subsets

is about 5 to 10 times less than complexity of the exhaustive search.

The complexity can be further reduced by noting that only the function $f()$ and $g()$ are dependent on the received signal value, namely $y$ (the rest is computed once for the entire frame.)

\section{Application in Space-Time coding}

In this part, we introduce a new structure for block spacetime code over two transmit antennas and two symbol periods. This space-time coding scheme is full rate, full diversity with high coding advantage. We will show that the problem of the decoding of the proposed method can be simplified in the same manner as described earlier for the case of the BLAST systems. The structure of this code for $r$-PSK modulation is as follows:

$$
C_{m, n}=A^{m}+D A^{n} \in \mathcal{C}^{2 \times 2} \quad m, n=0,1, \ldots, r-1
$$

where

$$
A=\left[\begin{array}{cc}
\theta_{1} & 0 \\
0 & \theta_{k}
\end{array}\right] \quad D=\left[\begin{array}{cc}
0 & \theta_{s} \\
1 & 0
\end{array}\right]
$$

and

$$
\theta_{n}=\exp \left(j \frac{2 \pi}{r} n\right)
$$

and $m$ and $n$ are the information symbols.

In table I, the best values for $k$ and $s$ for $r$-PSK $(4 \leq r \leq$ 32 ) to have the highest coding advantage are listed.

\begin{tabular}{|c|c|c|}
\hline & $k$ & $s$ \\
\hline \hline 4PSK & 1,3 & 1,3 \\
8PSK & 3 & $1,3,5,7$ \\
16PSK & 7 & $2,6,10,14$ \\
32PSK & 7,23 & $3,5,11,19,21,27,29$ \\
\hline
\end{tabular}

TABLE I

CODE STRUCTURE FOR $r$-PSK

Note that this code is a superposition of two matrices: $A^{m}$ and $D A^{n}$ that both of them form a finite group, similar to points of PSK constellations. As we will see latter, this similarity leads us to a similar decoding formulation of decoding.

Coding advantage is one of the well-known metrics to evaluate performance of the different space-time codes structures [2]. Simulations show significant improvement for the coding advantage of the proposed scheme as compared with the Alamouti code [4]. Also, the proposed scheme achieves a coding advantage very close to that of the Damen code [5]. Recall that the latter scheme uses an algebraic structure to construct non-orthogonal codes with large coding advantage. The decoding method of the Damen code is based on sphere decoding which is generally a complex operation.

As an example, in four bits throughput, the coding advantage of the proposed scheme is about $4.7 \mathrm{~dB}$ larger than that of the Alamouti code and is very close to the coding advantage of the Damen code. Figures 3 and 4 show the SER curves of the Alamouti code, the Damen code, and the proposed scheme for 3 and 4 bits throughput. These figures show more than $2 \mathrm{~dB}$ and $5 \mathrm{~dB}$ improvement in coding gain in three and four bits throughput, compared with the Alamouti code. Also, fig. 4 shows that the performance of the proposed scheme is very similar to the Damen code.

In the following, we show that the decoding problem of this scheme has the same formulation with the previous problem. Again, to formulate the $M L$ decoding for this code, we have,

$$
P\left(Y \mid H, C_{m, n}\right)=\frac{1}{\pi^{N T}} \exp \left(-d_{m n}^{2}\right)
$$

where $Y$ is the matrix corresponding to the received signal, and,

$$
d_{m n}^{2}=\operatorname{tr}\left[\left(Y-\sqrt{\frac{\rho}{M}} H C_{m, n}\right)\left(Y-\sqrt{\frac{\rho}{M}} H C_{m, n}\right)^{*}\right] .
$$

Now, let us redefine $K, f(n), g(m)$ and $h(m-n)$ as,

$$
\begin{gathered}
K=\operatorname{tr}\left\{Y Y^{*}+2 \frac{\rho}{2} H H^{*}\right\} \\
f(m)=-\sqrt{\frac{\rho}{2}} \operatorname{tr}\left\{H A^{m} Y^{*}+Y A^{-m} H^{*}\right\} \\
g(n)=-\sqrt{\frac{\rho}{2}} \operatorname{tr}\left\{H D A^{n} Y^{*}+Y A^{-n} D^{*} H^{*}\right\} \\
h(m-n)=\frac{\rho}{2} \operatorname{tr}\left\{H A^{m-n} D^{*} H^{*}+H D A^{n-m} H^{*}\right\} .
\end{gathered}
$$

Using these notations, we can show that,

$$
d_{m n}^{2}=K+f(m)+g(n)+h(m-n)
$$

Recall that $A^{r}=A^{0}=I_{2 \times 2}$, so similar to (9), $h(m-n)=$ $h(m-n \bmod r)$.

Comparing (9) and (23), it is easy to see that Euclidian distance in BLAST system and this structure of space-time code has the same format. As a result, the introduced decoding method is applicable for this space-time scheme as well. 


\section{CONCLUSION}

In this paper, a new low-complex ML decoding method for MIMO channels based on PSK constellation with two transmit antennas is introduced. The proposed method of decoding is applied for both BLAST system and a new full-rate fulldiversity space-time code.

We use the feature that the points of a PSK constellation form a finite group under complex multiplication to substantially reduce the decoding complexity. Also it is shown that similar ideas can be applied to other symmetric constellations by breaking down the constellation into a number of PSK-type subsets.

A new full diversity full rate block space-time code is introduced. This code is a superposition of two families of matrices, where each of them forms a finite group under complex multiplication. We show that the problem of the decoding of the proposed method can be simplified in the same manner as described earlier for the case of the BLAST systems. Simulations show significant improvement for the coding advantage of the proposed scheme as compared with Alamouti code. Also, the proposed scheme achieves a coding advantage very close to that of the Damen code. Recall that the latter scheme uses an algebraic structure to construct nonorthogonal codes with large coding advantage. The decoding method of the Damen code is based on the sphere decoding which is generally a complex operation.

\section{REFERENCES}

[1] G. J. Foschini, "Layered space-time architecture for wireless communication in a fading environment when using multi-element antennas," Bell Labs Tech. J., pp. 41-59, Autumn 1996.

[2] V. Tarokh, N. Seshadri and A. R. Calderbank, "Space-time code for high data rate wireless communication: Performance criterion and code construction," IEEE Trans. Inform. Theory, vol. 44, pp. 744-765, Mar. 1988.

[3] G. D. Golden P. W. Wolniansky, G. J. Foschini and R. A. Valenzuela, "V-BLAST: An architecture for realizing very high data rates over the rich-scattering wireless channel," in Proc. ISSSE-98, Pisa, Italy, Sept. 1999, vol. 3 .

[4] S. M. Alamouti, "A simple transmit diversity technique for wireless communication," IEEE J. Select. Areas Commun., vol. 16, pp. 14511458, Oct. 1998

[5] M. O. Damen, A. Tewfik, and J. C. Belfiore, "A construction of spacetime code based on number theory," IEEE Trans. Inform. Theory, vol. 48, pp. 753-760, Mar. 2002.

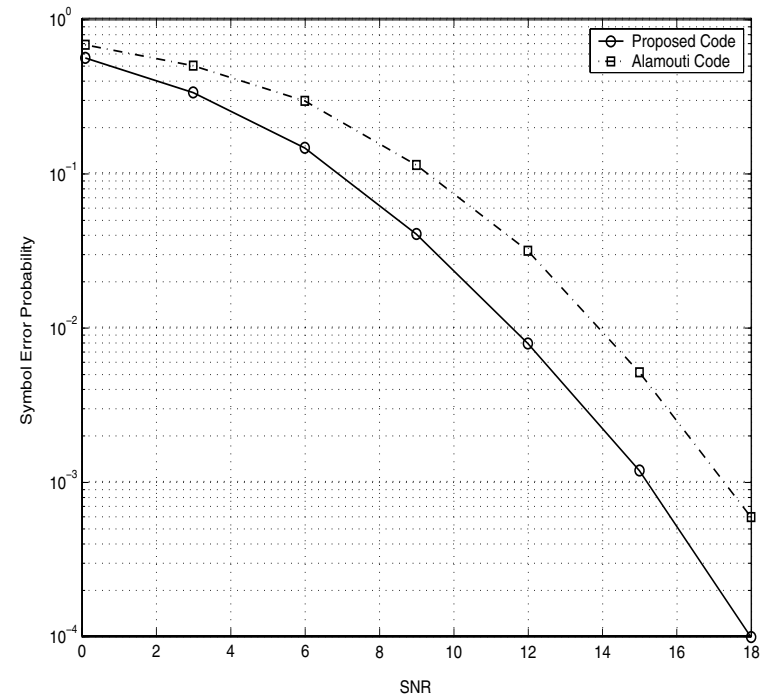

Fig. 3. Symbol-Error-Rate for two transmit and two receive antennas3 bit PCU

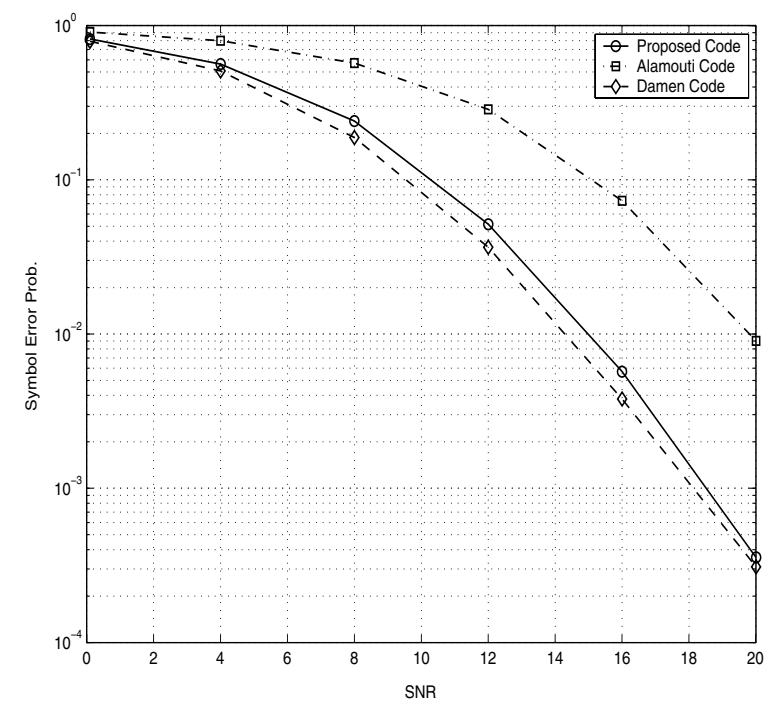

Fig. 4. Symbol-Error-Rate for two transmit and two receive antennas4 bit PCU 